\title{
Neuropsicologia do Transtorno de Déficit de Atenção/Hiperatividade: Modelos Neuropsicológicos e Resultados de Estudos Empíricos
}

\author{
Flávia Wagner - Universidade Federal do Rio Grande do Sul, Porto Alegre, Brasil \\ Luis Augusto de Rohde - Universidade Federal do Rio Grande do Sul, Porto Alegre, Brasil \\ Clarissa Marceli Trentini - Universidade Federal do Rio Grande do Sul, Porto Alegre, Brasil
}

\begin{abstract}
Resumo
Diferentes modelos teóricos foram propostos para explicar os déficits neuropsicológicos e seu impacto no comportamento de indivíduos com Transtorno de Déficit de Atenção/Hiperatividade (TDAH). Os objetivos deste artigo serão apresentar os principais modelos neuropsicológicos do TDAH e possibilitar uma atualização acerca dos principais achados relacionados ao funcionamento neuropsicológico de pacientes com esse diagnóstico. Revisões e meta-análises sobre funcionamento executivo, aversão à resposta tardia, processamento temporal e variações intraindividuais no tempo de reação são apresentadas. O perfil neuropsicológico de pacientes com TDAH é heterogêneo e testes neuropsicológicos não são suficientemente sensíveis e específicos para realização do diagnóstico, embora sejam muito importantes na identificação do perfil cognitivo para estabelecimento de um plano de tratamento mais abrangente e adequado às necessidades especificas de cada paciente.

Palavras-chave: transtorno de déficit de atenção/hiperatividade, neuropsicologia, revisão de literatura
\end{abstract}

\section{Neuropsychology of Attention-Deficit/Hyperactivity Disorder:}

Neuropsychological Models and Results of Empirical Studies

\begin{abstract}
Abstrat
Different theoretical models have been proposed to explain the neuropsychological deficits and their impact on the behavior of individuals with Attention-Deficit/ Hyperactivity Disorder (ADHD). The aims of this article will be to present the main neuropsychological models of ADHD and an update on the main findings related to neuropsychological functioning of patients with this diagnosis. Reviews and meta-analyzes on executive functioning, delay aversion, temporal processing and intra-individual variability in reaction time are presented. The neuropsychological profile of patients with ADHD is heterogeneous and neuropsychological tests are not sufficiently sensitive and specific for correct diagnosis, although they are very important to identify the cognitive profile and establish a more comprehensive and appropriate treatment plan according to the characteristics of each patient.

Keywords: attention-deficit/hyperactivity disorder, neuropsychology, literature review
\end{abstract} Neuropsicología del Trastorno de Déficit de Atención/Hiperactividad:
Modelos Neuropsicológicos y Resultados de Estudios Empíricos

\begin{abstract}
Resumen
Diferentes modelos teóricos han sido propuestos para explicar el déficit neuropsicológico y su impacto en el comportamiento de personas con Trastorno por Déficit de Atención/Hiperactividad (TDAH). Los objetivos de este artículo han sido presentar los principales modelos neuropsicológicos de TDAH y posibilitar una actualización sobre los principales resultados relacionados al funcionamiento neuropsicológico de pacientes con ese diagnóstico. Revisiones y meta- análisis sobre funcionamiento ejecutivo, aversión a la respuesta tardía, procesamiento temporal y variaciones interindividuales, en el tiempo de reacción se presentan. El perfil neuropsicológico de pacientes con TDAH es heterogéneo y los tests neuropsicológicos no son suficientemente sensibles y específicos para la realización del diagnóstico, aunque sean muy importantes para identificar el perfil cognitivo y establecer un plan de tratamiento más integral y adecuado a las necesidades específicas de cada paciente.

Palabras-clave: trastorno de déficit de atención/hiperactividad, neuropsicología, revisión de literatura
\end{abstract}

O Transtorno de Déficit de Atenção/Hiperatividade (TDAH) é um transtorno neurodesenvolvimental caracterizado pela presença de desatenção, hiperatividade e/ou impulsividade presentes em um nível mais frequente e grave do que aquele tipicamente observado em indivíduos em nível equivalente de desenvolvimento (American Psychiatric Association, 2013). A prevalência mundial é estimada em torno de 5\% (Polanczyk, de Lima, Horta, Biederman, \& Rohde, 2007) e os sintomas iniciam-se na infância e persistem na adolescência

Disponivel em wnw.scielo.br e na idade adulta em um número considerável dos casos (Biederman, 2005).

O diagnóstico de TDAH é essencialmente clínico, baseado em critérios estabelecidos em sistemas classificatórios como o DSM-5 (American Psychiatric Association, 2013) e a CID-10 (Organização Mundial da Saúde, 1993) (Rohde \& Halpern, 2004). De acordo com os parâmetros clínicos da Academia Americana de Psiquiatria da Criança e do Adolescente (Pliszka \& Aacap Work Group on Quality Issues, 2007), a avaliação do 
TDAH deve incluir entrevistas com pais ou responsáveis e com o próprio paciente, investigação acerca do funcionamento escolar, de comorbidades psiquiátricas e revisão do histórico médico, psicossocial e familiar. Escalas de avaliação do comportamento, preenchidas tanto por pais ou responsáveis quanto por professores, são ferramentas úteis para o processo de avaliação dos sintomas, embora não seja recomendado seu uso como única fonte para realização do diagnóstico. Ainda segundo os parâmetros clínicos da Academia Americana de Psiquiatria da Criança e do Adolescente, a utilização de testes psicológicos ou neuropsicológicos não é obrigatória para o diagnóstico do TDAH, mas é recomendada em casos de suspeita de déficits intelectuais ou transtornos de aprendizagem, podendo contribuir de forma significativa para o entendimento de déficits funcionais do paciente.

Apesar de não fazer parte dos critérios diagnósticos, o perfil de déficits neuropsicológicos desse transtorno tem sido amplamente investigado (Nigg, 2013). A busca pela elucidação dos aspectos neuropsicológicos relacionados ao TDAH está relacionada à investigação dos mecanismos neuropsicológicos ou biológicos que possam explicar o desenvolvimento e a expressão desse transtorno (Sonuga-Barke, 2005; Stefanatos \& Baron, 2007). Embora haja fortes evidências da influência de fatores genéticos, ambientais e biológicos na etiologia, a patofisiologia desse transtorno ainda não está completamente elucidada (Biederman, 2005). Nesse sentido, diferentes modelos teóricos foram propostos para explicar os déficits neuropsicológicos e seu impacto no comportamento de indivíduos com TDAH. Diante disso, este artigo terá por objetivo apresentar as principais contribuições do estudo da neuropsicologia do TDAH para a compreensão da patofisiologia desse transtorno. Para tanto, serão apresentados 1) os principais modelos neuropsicológicos do TDAH; 2) uma atualização acerca dos principais resultados empíricos relacionados à neuropsicologia do TDAH e 3) as implicações para a prática clínica.

\section{Modelos Neuropsicológicos do TDAH}

\section{Teoria da Autorregulação (Barkley, 1997, 1998)}

Barkley apresenta um modelo híbrido das funções pré-frontais, o qual considera um modelo neuropsicológico desenvolvimental da autorregulação humana. De acordo com essa teoria, a inibição comportamental representa o componente fundamental e permite o funcionamento das outras quatro funções executivas que compõem o modelo: 1) memória de trabalho; 2) autorregulação do afeto, motivação e excitação (arousal); 3) internalização do discurso; e 4) reconstituição.

A inibição comportamental refere-se a três processos inter-relacionados: 1) inibição de uma resposta inicial prepotente; 2) suspensão de uma resposta em andamento; 3) controle de interferência. Os dois primeiros processos permitem ao sistema um atraso na resposta, o qual possibilita a ação das outras quatro funções executivas. $\mathrm{O}$ controle de interferência garantirá que outros eventos concorrentes não rompam o período de atraso que possibilita a autorregulação. A autorregulação pode ser entendida como uma resposta do indivíduo que altera a probabilidade de uma consequência relacionada ao evento em andamento. Assim, a autorregulação gera uma lapso de tempo que possibilita a ação das outras quatro funções executivas que compõem o modelo.

Com relação à ação das demais funções executivas, a memória de trabalho possibilita ao indivíduo manter na mente informações representadas internamente (on-line) para que possam ser usadas para controlar uma resposta subsequente. A autorregulação do afeto, motivação e excitação (arousal) envolve a capacidade de adiar a expressão de reações emocionais que seriam eliciadas por um evento. Também diz respeito à habilidade de regular e até mesmo induzir estados emocionais e estados de excitação (arousal) necessários para a realização de um comportamento. A internalização do discurso permite descrição e reflexão, autoinstrução, autoquestionamento e resolução de problemas. Por fim, a reconstituição envolve processos de análise, síntese e simulação de um comportamento, fluência verbal e comportamental e criatividade dirigida a um objetivo.

Em conjunto, as funções executivas, que dependem da ativação da inibição comportamental, permitem ao indivíduo internalizar comportamentos para antecipar mudanças e suas consequências. Elas também produzem efeitos observáveis na resposta comportamental e no controle motor, minimizando ou suprimindo comportamentos não relacionados a um objetivo específico transferido ao sistema de execução motor. Assim, o indivíduo que apresenta déficits na inibição comportamental, irá apresentar dificuldades na inibição de respostas que são irrelevantes para uma tarefa em andamento, na flexibilidade comportamental, na sensibilidade a feedback, na persistência na realização de tarefas e na retomada de uma tarefa após interrupção. 
2. Modelo Cognitivo Energético (Sergeant, 2000, 2005; van der Meere, 2005)

O Modelo Cognitivo Energético (Cognitive Energetic Model - CEM) é uma tentativa de reunir processos top-down e bottom-up em uma mesma teoria. De acordo com esse modelo, o processamento da informação depende da relação entre três níveis de funcionamento: 1) mecanismos computacionais de processamento da informação; 2) fatores de estado (state factors) e 3) gerenciamento/funcionamento executivo. $\mathrm{O}$ nível de mecanismos computacionais envolve quatro estágios que ocorrem entre a apresentação do estímulo e a emissão da resposta: codificação (decodificação da informação), busca (busca de informações na memória para decidir qual será a resposta), decisão (qual resposta é a mais adequada) e organização motora (compatibilização da resposta e da preparação motora). A disponibilidade desses estágios está relacionada aos graus de ativação (activation), excitação (arousal) e esforço (effort) do sujeito, que fazem parte do segundo nível. Esforço é a energia necessária para realizar alguma tarefa, sendo necessário quando o estado atual do organismo não é o mesmo demandado pela tarefa. Está relacionado à demanda cognitiva de uma atividade e engloba fatores como motivação e resposta a contingências. O esforço interfere na ativação ou inibição dos conjuntos energéticos de excitação e ativação. Excitação é o nível de prontidão para agir em relação a um estímulo em um momento específico, sendo considerada, por isso, fásica. Assim, tende a ser influenciada pela intensidade e novidade do estímulo, podendo ser caracterizada pela pergunta "O que é isso?". A Ativação, por outro lado, é associada à prontidão fisiológica tônica para responder de forma voluntária a estímulos mais duradouros. Pode ser caracterizada pela pergunta “O que deve ser feito?". Por fim, o terceiro nível, associado ao córtex pré-frontal, engloba sistemas executivos de monitoramento, planejamento, detecção e correção de erros. De acordo com a essa teoria, indivíduos com TDAH poderiam ter déficits em qualquer um dos três níveis.

\section{Aversão à resposta Tardia (Delay Aversion) (Sonuga-Barke,} 2002)

Sonuga-Barke (2002) propôs um modelo associado aos mecanismos de recompensa. De acordo com esse modelo, os comportamentos característicos de indivíduos com TDAH seriam expressões de um estilo motivacional subjacente, em oposição à teoria de que seriam decorrentes de uma disfunção no controle inibitório. Assim, crianças com TDAH seriam motivadas a escapar ou evitar situações em que existe atraso na recompensa. Além de tender a escolher recompensas menores e imediatas, a aversão à resposta tardia também poderia manifestar-se por meio de aumento na atividade, atenção e frustração em momentos em que é necessário esperar (Sonuga-Barke, Sergeant, Nigg, \& Willcutt, 2008).

\section{Modelo de Múltiplos Caminhos (Sonuga-Barke, Bitsakou, \& Thompson, 2010)}

Os modelos de Autorregulação e de Aversão à Resposta Tardia propõem explicar os sintomas comportamentais de indivíduos com TDAH por meio de um déficit neuropsicológico ou processo neuropsicológico específico. Entretanto, os estudos realizados até o momento indicam que mais de um mecanismo neuropsicológico podem estar envolvidos na patofisiologia do TDAH, pois comparações do desempenho neuropsicológico entre grupos com e sem o transtorno podem eventualmente apresentar diferenças significativas, entretanto, o tamanho de efeito é geralmente modesto, indicando que os déficits estão presentes em apenas um subgrupo dos pacientes com TDAH (Nigg, 2005). Isso significa que nem todos os pacientes com o diagnóstico de TDAH apresentam o mesmo perfil de déficits neuropsicológicos e alguns podem não apresentar nenhum dos déficit descritos, ou pelo menos alterações em testes que se proponham a medir esses constructos.

Assim, essa limitação dos modelos até então propostos e a heterogeneidade em relação aos déficits neuropsicológicos de indivíduos com TDAH levaram à mudança da busca por um único e principal déficit para a identificação de múltiplos caminhos desenvolvimentais nos quais os diferentes déficits podem ser considerados complementares ao invés de competitivos (Sonuga-Barke, 2005). Propôs-se um modelo dual, no qual déficits no controle inibitório e nos mecanismos de recompensa comporiam dois subtipos distintos do transtorno (Sonuga-Barke, 2002). O caminho associado ao controle inibitório deficiente estaria associado ao córtex pré-frontal enquanto o caminho associado à aversão à resposta tardia estaria associado ao núcleo acumbens. Os caminhos estariam associados a diferentes sintomas, perfis cognitivos e motivacionais e origens genéticas e não genéticas. A formulação mais recente da teoria de múltiplos caminhos envolve um terceiro componente, a percepção temporal (Sonuga-Barke, Bitsakou, \& Thompson, 2010). A percepção temporal envolve déficits na discriminação de intervalos de 
tempo e manutenção de um ritmo motor constante (Noreika, Falter, \& Rubia, 2013).

\section{Principais Resultados de Pesquisas Empiricas e Meta-Análises}

O desenvolvimento dos Modelos Neuropsicológicos do TDAH proporcionaram um avanço importante do entendimento do transtorno ao longo das últimas décadas. Isso ocorreu, em especial, por meio do reconhecimento de que múltiplas redes neurais estão envolvidas no transtorno e de que muitos dos déficits estudados não estão presentes em todos os indivíduos com TDAH (Nigg, 2005). Além disso, um maior conhecimento acerca dos mecanismos neurobiológicos tem possibilitado a exploração de outras funções neuropsicológicas e, consequentemente, a proposição ou revisão de modelos que deem conta da heterogeneidade da expressão fenotípica do TDAH, como o Modelo de Múltiplos Caminhos. Assim, nesta segunda seção, serão apresentados os principais resultados de pesquisas empíricas relacionadas à neuropsicologia do TDAH, considerando os principais construtos relevantes para os Modelos Neuropsicológicos citados. É importante ressaltar que um mesmo construto pode estar relacionado ou ser explicado a partir de mais de uma teoria, como é o caso do Funcionamento Executivo, por exemplo. Além disso, muitos dos estudos citados não tiveram como base os modelos já apresentados, mas basearam-se em características comportamentais ou mecanismos neurobiológicos reconhecidamente associados ao transtorno. Por fim, tendo em vista a diversidade de testes ou tarefas neuropsicológicas utilizados na avaliação dessas funções, essa seção irá apresentar, sempre que possível, resultados de revisões e meta-análises.

\section{Funções Executivas}

O termo "Funções Executivas" (FE) engloba um conjunto de capacidades cognitivas responsáveis por processos como planejamento, inibição, sequenciamento, e monitoramento de comportamentos complexos. Disfunções executivas têm sido associadas com lesões no córtex frontal e suas conexões com tálamo e núcleos da base (Royall et al., 2002). Ardila (Ardila, 2008) propõe duas formas relacionadas de FE: A primeira, que denomina de FE metacognitivas, envolve resolução de problemas, memória de trabalho, planejamento, controle atencional, entre outros e está relacionada a áreas pré-frontais dorsolaterais. A segunda, FE emocionais/motivacionais, está relacionada à coordenação entre cognição e emoção/ motivação e está associada a áreas frontais mediais e órbito-frontais.

O funcionamento deficiente do córtex frontal está relacionado com características comportamentais consistentes com o perfil do TDAH, tais como dificuldade de sustentar a atenção em tarefas complexas, falta de flexibilidade cognitiva e ineficiência em processar rapidamente novas informações (Nigg \& Casey, 2005). Apesar de déficits em funções executivas estarem associados ao TDAH, eles não fazem parte do critério diagnóstico e indivíduos com o transtorno podem não apresentar nenhum déficit clinicamente observável (Sonuga-Barke et al., 2008).

Uma meta-análise de 29 estudos que utilizaram a tarefa de controle inibitório Stop Task foi realizada (Lijffijt, Kenemans, Verbaten, \& van Engeland, 2005). O Stop Task é uma tarefa computadorizada na qual os participantes devem responder o mais rápido possível aos estímulos (go stimulus), mas devem inibir respostas quando um segundo estímulo de parada (stop stimulus) é apresentado logo após o aparecimento do go stimulus (geralmente após 100-500ms). Esse estímulo de parada avalia a capacidade de o participante inibir uma resposta em andamento, capacidade que, de acordo com a Teoria da Autorregulação, estaria prejudicada em indivíduos com TDAH. Pode-se avaliar tanto o tempo de reação total e a acurácia, quanto o tempo de reação ao sinal de parada (stop signal reaction time - SSRT). Os resultados da meta-análise indicam diferenças com tamanhos de efeito médio entre crianças e adolescentes com e sem TDAH no SSRT. Além disso, observou-se um tempo de reação total médio mais longo em indivíduos com o transtorno bem como uma maior variabilidade no tempo de reação, variável que está mais relacionada à capacidade de vigilância e ao conjunto de ativação, segundo a teoria dos fatores de estado.

Uma meta-análise de 83 estudos que utilizaram medidas de FE em grupos com e sem TDAH foi conduzida (Willcutt, Doyle, Nigg, Faraone, \& Pennington, 2005). Os resultados mais consistentes foram encontrados para controle inibitório, medido pelo stop signal reaction time, e vigilância, avaliado através do número de erros por omissão no Continuous Performance Test. Também houve diferenças significativas em medidas de planejamento, mensuradas através de testes como a Torre de Hanói, Torre de Londres, Labirintos de Porteus e a Figura Complexa de Rey, sendo os resultados mais consistentes para os testes Torre de Hanói e Labirintos de Porteus. Os resultados também indicam dificuldades em tarefas de memória de trabalho verbal e não 
verbal, sendo que os maiores tamanhos de efeito foram encontrados em tarefas não verbais. Especificamente em relação à memória de trabalho, uma meta-análise de 26 estudos corroborou os achados de (Willcutt et al., 2005), identificando déficits tanto na memória de trabalho verbal quanto espacial, embora com tamanhos de efeito maiores para memória de trabalho não verbal (Martinussen, Hayden, Hogg-Johnson, \& Tannock, 2005).

A meta-análise de (Willcutt et al., 2005) também indica que os resultados do Teste Wisconsin de Classificação de Cartas, especificamente do escore de erros perseverativos, apresentam menor capacidade de detectar diferenças entre grupos. Outra meta-análise investigou 17 estudos com o teste Stroop e evidenciou que indivíduos com TDAH apresentam um desempenho médio mais prejudicado nas medidas de leitura de palavras, nomeação de cores e no escore de interferência. Porém, o tamanho de efeito foi modesto e bastante heterogêneo entre os estudos, sendo próximo de $0 \mathrm{em}$ alguns deles. Os autores destacam que o método utilizado para calcular o escore de interferência influencia significativamente os resultados e discutem se o Teste Stroop, em seus formatos atuais, constitui uma medida válida de controle de interferência (van Mourik, Oosterlaan, \& Sergeant, 2005).

Uma questão importante em relação ao funcionamento executivo diz respeito à validade ecológica dos testes disponíveis. Déficits encontrados em testes que se propõem a medir tais construtos não necessariamente refletem dificuldades observadas em atividades da vida real (Jurado \& Rosselli, 2007). Escalas ou questionários baseados em comportamentos associados ao funcionamento executivo não apresentam resultados semelhantes àqueles obtidos através de testes de desempenho. Uma revisão de 20 estudos com amostras de crianças e adultos analisou a associação entre o desempenho executivo medido através de testes e de questionários. Os resultados indicaram uma correlação baixa entre esses dois tipos de medida, sugerindo que estejam medindo diferentes aspectos do construto (Toplak, West, \& Stanovich, 2013). Assim, deve-se considerar que os resultados observados nos testes psicológicos que avaliam funcionamento executivo podem estar refletindo o funcionamento de pacientes em tarefas que não necessariamente são relacionadas ou interferem diretamente nas atividades da vida diária dos pacientes.

Além da validade ecológica, ainda é importante destacar que que os déficits em funções executivas não são exclusivos em TDAH e estão presentes em vários outros transtornos psiquiátricos, como Transtornos de Humor, Autismo e Transtornos de Aprendizagem. Além disso, déficits em outras funções cognitivas não relacionadas ao funcionamento executivo, tal como variabilidade de tempo de reação, diferenciam pacientes com e sem TDAH com tamanhos de efeito tão grande quanto ou superiores aos reportados para as medidas de FE (Nigg, 2010; Willcutt, Sonuga-Barke, Nigg, \& Sergeant, 2008).

\section{Aversão à Resposta Tardia (ART)}

A Aversão à Resposta Tardia é geralmente medida através de paradigmas experimentais de escolha-atraso (choice-delay), como o Choice Delay Task (Sonuga-Barke, Taylor, Sembi, \& Smith, 1992), o Maudsley Index of Childhood Delay Aversion Task (MIDA) (Kuntsi, Oosterlaan, \& Stevenson, 2001) e o Iowa Gambling Task (IGT) (Bechara, Damasio, Damasio, \& Anderson, 1994). Nas duas primeiras tarefas, o experimento consiste na escolha entre um prêmio maior que exige um tempo maior de espera ou um prêmio menor e imediato. Nesse tipo de tarefa, geralmente há recompensas reais, como um valor em dinheiro ou algum objeto que o participante recebe conforme sua pontuação. Tanto a tarefa MIDA quanto a Choice Delay Task diferenciam indivíduos com e sem TDAH, com tamanhos de efeito moderados, semelhantes aos observados para tarefas de controle inibitório e memória de trabalho (Sonuga-Barke et al., 2008).

O IGT (Bechara et al., 1994) foi desenvolvido para avaliar e quantificar deficiências na tomada de decisão de pacientes neurológicos, simulando decisões da vida real em condições que incluem recompensas, punições e incertezas. Um estudo com uma versão adaptada do IGT não encontrou diferenças significativas entre pacientes com TDAH e controles. No entanto, os autores ressaltam que crianças com TDAH tendem a apresentar um padrão de respostas mais inconsistentes nas escolhas desvantajosas além de maior perseveração nas respostas se comparado aos controles (Geurts, van der Oord, \& Crone, 2006). O desempenho de adolescentes com TDAH através do IGT também foi analisado e os resultados indicaram que pacientes com o transtorno selecionam cartas menos vantajosas se comparados aos controles (Toplak, Jain, \& Tannock, 2005). Assim como o estudo anterior (Geurts et al., 2006), esse estudo demonstrou que os resultados do IGT não estão relacionados a tarefas de funções executivas, corroborando a hipótese de múltiplos caminhos. 
O papel do reforço comportamental em tarefas neuropsicológicas variadas, como Stop Task, Tarefa de Aritmética, Choice Delay Task, entre outras foi analisado através de 22 estudos (Luman, Oosterlaan, \& Sergeant, 2005). Em relação ao reforço, diferentes combinações de contingências foram analisadas: presença de recompensa, ausência de recompensa, custo da resposta e feedback em relação ao desempenho. Também foram consideradas recompensas parciais e intensidade $\mathrm{da}$ recompensa. Os resultados indicam que o reforço tem um efeito positivo no desempenho das tarefas analisadas e no nível de motivação para crianças com e sem TDAH. No entanto, o efeito do reforço tende a ser mais eficaz no desempenho de crianças com TDAH, além de que reforços mais intensos tendem a ser mais eficazes nesse grupo.

\section{Déficits no Processamento Temporal}

O processamento temporal é a habilidade de perceber e representar o tempo e permite a organização sequencial de eventos e ações, além de antecipar ou predizer quando eventos futuros irão ocorrer. Por ser um construto multidimensional, uma variedade de métodos pode ser utilizada para mensurá-lo, dificultando a compreensão da extensão em que pacientes com TDAH apresentam algum déficit. Os principais processos avaliados são a habilidade de discriminar intervalos de tempo que diferem em milissegundos ou segundos (duration discrimination), julgamento do intervalo de tempo que separa dois estímulos (temporal judgment), reprodução de intervalos de tempo com precisão (duration reproduction), previsão de tempo em que um estímulo irá ocorrer (anticipation) e resposta motora seguindo um ritmo determinado (tapping). Os principais déficits associados ao TDAH são relacionados à discriminação e reprodução de intervalos de tempo e à resposta motora ritmada (Toplak, Dockstader, \& Tannock, 2006). O processamento da informação temporal envolve a integração de circuitos corticais com os gânglios da base, cerebelo e hipocampo (Meck, 2005). O córtex pré-frontal também tem um papel importante, especialmente em intervalos longos, que além do processamento temporal, também requerem atenção sustentada ou memória de trabalho (Toplak et al., 2006).

Variabilidade intraindividual no tempo de reação (VII) e Negociação entre Velocidade e Acurácia

Uma meta-análise de 283 estudos indica uma diferença significativa na VII do tempo de reação de pacientes com TDAH quando comparados a controles com desenvolvimento típico, com tamanhos de efeito de magnitude média a grande. Os resultados dessa meta-análise também indicam que, ao contrário do que é reportado em estudos anteriores, quando considerada a maior variabilidade, pacientes com TDAH não apresentam tempos de reação mais longos (Kofler et al., 2013). As diferenças entre TDAH e controles na VII no tempo de reação tendem a diminuir ou desaparecer quando a apresentação dos estímulos é mais rápida ou quando há recompensas, sugerindo que déficits energéticos possam estar associados (Kuntsi \& Klein, 2012). Assim, esse resultado pode ser interpretado como um déficit no fator de estado "excitação" do Modelo Cognitivo Energético, que se refere à prontidão ou capacidade do indivíduo de manter-se alerta para responder aos estímulos (Huang-Pollock, Karalunas, Tam, \& Moore, 2012). Entretanto, esse déficit não é específico do TDAH, estando presente em outros transtornos psiquiátricos como transtornos do humor, de ansiedade, do espectro autista e disruptivos, bem como esquizofrenia e demência (Kofler et al., 2013; Tamm et al., 2012; Willcutt et al., 2008). As investigações em relação às bases neurológicas desse construto indicam um papel importante dos sistemas frontoestriatais (Kuntsi \& Klein, 2012).

A VII no tempo de reação é geralmente medida através do desvio-padrão do tempo de reação, embora existam outras formas como as análises Gaussianas Exponenciais, o Coeficiente de Variação e a Análise de Fourier (Kuntsi \& Klein, 2012). No entanto, tais análises não consideram, em um mesmo modelo, os resultados de velocidade e acurácia ou, como é denominada na literatura, a Negociação entre Velocidade e Acurácia. O Modelo de Difusão é uma derivação matemática que propõem utilizar variáveis observáveis (acurácia, tempo de reação médio e distribuição do tempo de reação) para calcular três parâmetros que refletem o processamento cognitivo: drift rate (velocidade/qualidade de acumulação da informação), boundary separation (evidência necessária para tomar uma decisão) e nondecision time (tempo utilizado em processos como decodificação e preparação motora e que não fazem parte do processo decisório) (Ratcliff \& McKoon, 2008). Uma meta-análise de 12 estudos que utilizaram uma tarefa baseada no paradigma Continuous Performance Test (CPT) indica que pacientes com TDAH tendem a ser mais lentos na velocidade de acumulação da informação (drift rate) do que controles sem TDAH (Huang-Pollock et al., 2012). Outra meta-análise de 5 estudos que compararam participantes com e sem TDAH e utilizaram ao 
menos um parâmetro do Modelo de Difusão indica diferenças significativas no drift rate, com tamanho de efeito médio a grande, além de nondecision times mais rápidos, com tamanho de efeito pequeno (Karalunas, Geurts, Konrad, Bender, \& Nigg, 2014). Embora ainda haja poucos estudos que utilizaram esse Modelo, os resultados tendem a indicar uma menor velocidade e qualidade na acumulação de informação em pacientes com TDAH. De acordo com o Modelo Cognitivo Energético, esse déficit pode estar relacionado com uma falha na alocação do fator de estado Esforço (Effort), ou seja, uma dificuldade de alocar a quantidade de energia necessária para realização da tarefa (Huang-Pollock et al., 2012).

\section{Limitações em Relação aos Estudos de Neuropsicologia do Tdah}

Embora tenha havido um avanço no entendimento da neuropsicologia do TDAH, alguns pontos necessitam ser observados. A maior parte das investigações em relação a aspectos neuropsicológicos do TDAH foi realizada considerando a apresentação Combinada. Além disso, poucos estudos analisam a apresentação Hiperativa-Impulsiva, especialmente devido à sua baixa prevalência na população se comparada às demais apresentações (Stefanatos \& Baron, 2007).

Com relação aos testes e tarefas neuropsicológicos, ressalta-se a necessidade de uma definição mais precisa dos construtos e o desenvolvimento de medidas com evidências de validade e fidedignidade. Também se deve ter em mente que os construtos de interesse para o TDAH são multifacetados e que o desempenho em uma tarefa tem múltiplos determinantes (Nigg, 2006; Stefanatos \& Baron, 2007). Além disso, testes neuropsicológicos de FE metacognitivas também dependem de FE emocianais/motivacionais, por isso apresentam validade ecológica limitada (Ardila, 2008). Ainda com relação à validade ecológica dos instrumentos neuropsicológicos, é necessário atentar para o fato de que as observações clínicas do comportamento e o relato de pais e professores não necessariamente estão correlacionados com os resultados de testes obtidos em um ambiente controlado de avaliação, dificultando a generalização para o mundo real (Stefanatos \& Baron, 2007).

Outra questão que merece atenção é a presença de diagnósticos comórbidos. Em indivíduos com o diagnóstico de TDAH, uma alta prevalência de outros diagnósticos é observada em contextos clínicos. $\mathrm{Na}$ população geral, aproximadamente metade dos indivíduos com TDAH Combinado apresenta comorbidade com Transtorno Opositor Desafiante e um quarto apresenta Transtorno de Conduta. Transtornos de Aprendizagem também são uma comorbidade frequente e Transtornos de Ansiedade e Depressão Maior ocorrem com mais frequência em pessoas com TDAH do que na população geral (American Psychiatric Association, 2013). Essa realidade clinica torna difícil a determinação de quanto as alterações encontradas são realmente associadas ao TDAH ou às condições comórbidas.

\section{Considerações Finais}

O desempenho de indivíduos com TDAH em tarefas neuropsicológicas tem sido amplamente investigado e permitiu que o conhecimento a respeito da neuropsicologia relacionada a esse transtorno tenha avançado muito nos últimos anos. A contribuição desse campo de conhecimento objetivou 1) a identificação de déficits neuropsicológicos específicos ao TDAH e 2) a construção de modelos teóricos que pudessem auxiliar na compreensão da patofisiologia desse transtorno, ou seja, na compreensão de seus mecanismos causais. Os avanços realizados até o momento, entretanto, demonstraram que nenhum construto neuropsicológico já estudado está deficitário em todos os indivíduos com TDAH e, até mesmo, alguns indivíduos com o transtorno não apresentam nenhum déficit neuropsicológico considerando os instrumentos e construtos já estudados. Esse resultado traz uma importante implicação para a pesquisa e para a prática clínica: a apresentação fenotípica do TDAH é heterogênea. $\mathrm{E}$ a heterogeneidade não está relacionada apenas a suas características neuropsicológicas, mas também clínicas/sintomatológicas, neurobiológicas e genéticas (Costa-Dias et al., 2013). Considerando essa heterogeneidade, a avaliação neuropsicológica tem um importante papel na caracterização dos pacientes que apresentam o transtorno, permitindo estabelecer forças e fraquezas no funcionamento cognitivo e auxiliando na detecção de diagnósticos comórbidos. Embora os escores de testes neuropsicológicos não sejam suficientemente sensíveis e específicos para realização do diagnóstico do transtorno (Nigg, 2013), o processo de avaliação neuropsicológica somado à avaliação clínica pode ter um papel fundamental na determinação e desenvolvimento de alvos de tratamento e monitoramento do progresso do paciente (Canadian Attention Deficit Hyperactivity Disorder Resource Alliance, 2011; Stefanatos \& Baron, 2007). 


\section{Referências}

American Psychiatric Association. (2013). Diagnostic and Statistical Manual of Mental Disorders (Fifth ed.). Arligton, VA: American Psychiatric Publishing.

Ardila, A. (2008). On the evolutionary origins of executive functions. Brain and Cognition, 68(1), 92-99.

Barkley, R. A. (1997). Behavioral inhibition, sustained attention, and executive functions: constructing a unifying theory of ADHD. Psychological Bulletin, 121, 65-94.

Barkley, R. A. (1998). Attention-Deficit Hyperactivity Disorder. A handbook for diagnosis and treatment. (2nd ed.). New York, NY: The Guilford Press.

Bechara, A., Damasio, A. R., Damasio, H., \& Anderson, S. W. (1994). Insensitivity to future consequences following damage to human prefrontal cortex. Cognition, 50(1-3), 7-15.

Biederman, J. (2005). Attention-deficit/hyperactivity disorder: a selective overview. Biological Psychiatry, 57, 1215-1220.

Canadian Attention Deficit Hyperactivity Disorder Resource Alliance. (2011). Canadian ADHD Practice Guidelines (Third ed.). Toronto ON: CADDRA.

Costa-Dias, T., Kieling, C., Graeff-Martins, A. S., Moriyama, T. S., Rohde, L. A., \& Polanczyk, G. V. (2013). Developments and challenges in the diagnosis and treatment of ADHD. Revista Brasileira de Psiquiatria, 35(1), S40-S50.

Geurts, H. M., van der Oord, S., \& Crone, E. A. (2006). Hot and cool aspects of cognitive control in children with ADHD: decision-making and inhibition. Journal of Abnormal Child Psychology, 34(6), 813-824.

Huang-Pollock, C. L., Karalunas, S. L., Tam, H., \& Moore, A. N. (2012). Evaluating vigilance deficits in ADHD: A meta-analysis of CPT performance. Journal of Abnormal Child Psychology, 121(2), 360-371.

Jurado, M. B., \& Rosselli, M. (2007). The elusive nature of executive functions: a review of our current understanding. Neuropsychology Review, 17(3), 213-233.

Karalunas, S. L., Geurts, H. M., Konrad, K., Bender, S., \& Nigg, J. T. (2014). Annual research review: Reaction time variability in ADHD and autism spectrum disorders: Measurement and mechanisms of a proposed trans-diagnostic phenotype.
Journal of Child Psychology and Psychiatry, 55(6), 685-710.

Kofler, M. J., Rapport, M. D., Sarver, D. E., Raiker, J. S., Orban, S. A., Friedman, L. M., \& Kolomeyer, E. G. (2013). Reaction time variability in ADHD: A meta-analytic review of 319 studies. Clinical Psychology Review, 33(6), 795-811.

Kuntsi, J., \& Klein, C. (2012). Intraindividual variability in ADHD and its implications for research of causal links. Current Topics in Behavioral Neurosciences, 9, 67-91.

Kuntsi, J., Oosterlaan, J., \& Stevenson, J. (2001). Psychological mechanisms in hyperactivity: I. Response inhibition deficit, working memory impairment, delay aversion, or something else? Journal of Child Psychology and Psychiatry, 42(2), 199-210.

Lijffijt, M., Kenemans, J. L., Verbaten, M. N., \& van Engeland, H. (2005). A meta-analytic review of stopping performance in attention-deficit/ hyperactivity disorder: deficient inhibitory motor control? Journal of Abnormal Psychology, 114(2), 216-222.

Luman, M., Oosterlaan, J., \& Sergeant, J. A. (2005). The impact of reinforcement contingencies on $\mathrm{AD} /$ HD: A review and theoretical appraisal. Clinical Psychology Review, 25(2), 183-213.

Martinussen, R., Hayden, J., Hogg-Johnson, S., \& Tannock, R. (2005). A meta-analysis of working memory impairments in children with attention-deficit/hyperactivity disorder. Journal of the American Academy of Child \& Adolescent Psychiatry, 44(4), 377-384.

Meck, W. H. (2005). Neuropsychology of timing and time perception. Brain and Cognition, 58(1), 1-8. doi: 10.1016/j.bandc.2004.09.004

Nigg, J. T. (2005). Neuropsychologic theory and findings in attention-deficit/hyperactivity disorder: The state of the field and salient challenges for the coming decade. Biological Psychiatry, 57, 1424-1435.

Nigg, J. T. (2010). Attention-Deficit/Hyperactivity DisorderEndophenotypes, Structure, and Etiological Pathways. Current Directions in Psychological Science, 19, 24-29.

Nigg, J. T. (2013). Attention deficits and hyperactivity-impulsivity: What have we learned, what next? Development and Psychopathology, 25(4), 1489-1503. 
Nigg, J. T., \& Casey, B. J. (2005). An integrative theory of attention-deficit/ hyperactivity disorder based on the cognitive and affective neurosciences. Development and Psychopathololy, 17, 785-806.

Noreika, V., Falter, C. M., \& Rubia, K. (2013). Timing deficits in attention-deficit/hyperactivity disorder (ADHD): Evidence from neurocognitive and neuroimaging studies. Neuropsychologia, 51(2), 235-266.

Organização Mundial da Saúde. (1993). Classificação dos Transtornos Mentais e de Comportamento da CID-10. Porto Alegre: Artes Médicas.

Pliszka, S., \& Aacap Work Group on Quality Issues. (2007). Practice parameter for the assessment and treatment of children and adolescents with attention-deficit/hyperactivity disorder. Journal of the American Academy of Child \& Adolescent Psychiatry, 46(7), 894-921.

Polanczyk, G., de Lima, M. S., Horta, B. L., Biederman, J., \& Rohde, L. A. (2007). The worldwide prevalence of ADHD: A systematic review and metaregression analysis. American Journal of Psychiatry, 164, 942-948.

Ratcliff, R., \& McKoon, G. (2008). The diffusion decision model: Theory and data for two-choice decision tasks. Neural Computing \& Applications, 20(4), 873-922.

Rohde, L. A., \& Halpern, R. (2004). Transtorno de déficit de atenção/hiperatividade: Atualização. Jornal de Pediatria, 80, S61-S70.

Royall, D. R., Lauterbach, E. C., Cummings, J. L., Reeve, A., Rummans, T. A., Kaufer, D. I., .. . Coffey, C. E. (2002). Executive control function: A review of its promise and challenges for clinical research. A report from the Committee on Research of the American Neuropsychiatric Association. Journal of Neuropsychiatry and Clinical Neurosciences, 14, 377-405.

Sergeant, J. A. (2000). The cognitive-energetic model: An empirical approach to attention-deficit hyperactivity disorder. Neuroscience \& Biobehavioral Reviews, 24(1), 7-12.

Sergeant, J. A. (2005). Modeling attention-deficit/ hyperactivity disorder: A critical appraisal of the cognitive-energetic model. Biological Psychiatry, 57(11), 1248-1255.

Sonuga-Barke, E. J. (2002). Psychological heterogeneity in $\mathrm{AD} / \mathrm{HD}$ : A dual pathway model of behaviour and cognition. Behavioural Brain Research, 130(1-2), 29-36.

Sonuga-Barke, E. J. (2005). Causal models of attentiondeficit/hyperactivity disorder: From common simple deficits to multiple developmental pathways. Biological Psychiatry, 57, 1231-1238.

Sonuga-Barke, E. J., Bitsakou, P., \& Thompson, M. (2010). Beyond the dual pathway model: Evidence for the dissociation of timing, inhibitory, and delay-related impairments in attention-deficit/hyperactivity disorder. Journal of the American Academy of Child and Adolescent Psychiatry, 49, 345-355.

Sonuga-Barke, E. J., Sergeant, J. A., Nigg, J., \& Willcutt, E. (2008). Executive dysfunction and delay aversion in attention deficit hyperactivity disorder: Nosologic and diagnostic implications. Child and Adolescent Psychiatric Clinics of North America, 17(2), 367-384.

Sonuga-Barke, E. J., Taylor, E., Sembi, S., \& Smith, J. (1992). Hyperactivity and delay aversion-I. The effect of delay on choice. Journal of Child Psychology and Psychiatry, 33(2), 387-398.

Stefanatos, G. A., \& Baron, I. S. (2007). Attention-deficit/hyperactivity disorder: A neuropsychological perspective towards DSM-V. Neuropsychology Review, 17(1), 5-38.

Tamm, L., Narad, M. E., Antonini, T. N., O’Brien, K. M., Hawk, L. W., \& Epstein, J. N. (2012). Reaction time variability in ADHD: A review. Neurotherapeutics, 9, 500-508.

Toplak, M. E., Dockstader, C., \& Tannock, R. (2006). Temporal information processing in ADHD: Findings to date and new methods. Journal of Neuroscience Methods, 151(1), 15-29.

Toplak, M. E., Jain, U., \& Tannock, R. (2005). Executive and motivational processes in adolescents with Attention-Deficit-Hyperactivity Disorder (ADHD). Behavioral and Brain Functions, 1(1), 8.

Toplak, M. E., West, R. F., \& Stanovich, K. E. (2013). Practitioner review: Do performance-based measures and ratings of executive function assess the same construct? Journal of Child Psychology and Psychiatry, 54(2), 131-143.

van der Meere, J. (2005). State Regulation and Attention Deficit Hyperactivity Disorder. In D. Gozal \& D. L. Molfese (Eds.), Attention Deficit 
Hyperactivity Disorder: From Genes to Patients (pp. 413433). Totowa, NJ: Humana Press Inc.

van Mourik, R., Oosterlaan, J., \& Sergeant, J. A. (2005). The Stroop revisited: A meta-analysis of interference control in AD/HD. Journal of Child Psychology and Psychiatry, 46(2), 150-165.

Willcutt, E. G., Doyle, A. E., Nigg, J. T., Faraone, S. V., \& Pennington, B. F. (2005). Validity of the executive function theory of attention-deficit/hyperactivity disorder: A meta-analytic review. Biological Psychiatry, 57, 1336-1346.
Willcutt, E. G., Sonuga-Barke, E. J. S., Nigg, J. T., \& Sergeant, J. A. (2008). Recent developments in neuropsychological models of childhood psychiatric disorders. In T. Banaschewski \& L. A. Rohde (Eds.), Advances in Biological Psychiatry. Biological Child Psychiatry. Recent Trends and Developments. (Vol. 24, pp. 195-226). Basel, Switzerland: Karger.

Recebido 06/06/2015

Reformulado 06/10/2015

Aceito 29/10/2015

Sobre os autores:

Flávia Wagner é psicóloga, especialista em Avaliação Psicológica e doutora em Psicologia pela Universidade Federal do Rio Grande do Sul (UFRGS). Atualmente é psicóloga da UFRGS e pesquisadora do Programa de Déficit de Atenção/Hiperatividade no Hospital de Clínicas de Porto Alegre.

E-mail: flavia_scs@yahoo.com.br

Clarissa Marceli Trentini é psicóloga, especialista em Avaliação Psicológica e doutora em Ciências Médicas: Psiquiatria pela Universidade Federal do Rio Grande do Sul (UFRGS). Atualmente é professora associada nível I do Instituto de Psicologia da UFRGS e professora dos cursos de graduação e pós-graduação em Psicologia.

E-mail: clarissatrentini@terra.com.br

Luis Augusto Rohde é psiquiatra, professor titular do Departamento de Psiquiatria e Medicina Legal da Universidade Federal do Rio Grande do Sul, diretor do Programa de Déficit de Atenção/Hiperatividade no Hospital de Clínicas de Porto Alegre e vice-coordenador do Instituto Nacional de Psiquiatria do Desenvolvimento.

E-mail:1rohde@terra.com.br

Contato com os autores:

Rua Ramiro Barcelos, 2350, sala 2201

CEP: 90035-903

Porto Alegre-RS, Brasil 